\title{
Epilepsy in Rett syndrome: Clinical and genetic features
}

\author{
Maria Pintaudi a,*, Maria Grazia Calevo ${ }^{\mathrm{b}}$, Aglaia Vignoli ${ }^{\mathrm{c}}$, Elena Parodi ${ }^{\mathrm{a}}$, Francesca Aiello ${ }^{\mathrm{a}}$, \\ Maria Giuseppina Baglietto ${ }^{a}$, Yussef Hayek ${ }^{\mathrm{d}}$, Sabrina Buoni ${ }^{\mathrm{d}}$, Alessandra Renieri ${ }^{\mathrm{e}}$, Silvia Russo ${ }^{\mathrm{f}}$, \\ Francesca Cogliati ${ }^{\mathrm{f}}$, Lucio Giordano ${ }^{\mathrm{g}}$, MariaPaola Canevini ${ }^{\mathrm{c}}$, Edvige Veneselli ${ }^{\mathrm{a}}$ \\ a Department of Child Neuropsychiatry, Epilepsy Centre, G. Gaslini Institute, University of Genoa, Genoa, Italy \\ ${ }^{\mathrm{b}}$ Epidemiology and Biostatistics Service, Scientific Directorate, IRCCS G. Gaslini, Genoa, Italy \\ c Epilepsy Centre, St. Paolo Hospital, Milan, Italy \\ d Pediatric Neuropsychiatric Unit, University Hospital, Policlinico Le Scotte, Siena, Italy \\ e Medical Genetics, Molecular Biology Department, University of Siena, Siena, Italy \\ ${ }^{\mathrm{f}}$ Department of Genetics, Istituto Auxologico Italiano, IRCCS, Milan, Italy \\ ${ }^{g}$ Pediatric Neuropsychiatric Division, City Hospital of Brescia, Brescia, Italy
}

\section{A R T I C L E I N F O}

\section{Article history:}

Received 7 June 2010

Received in revised form 25 June 2010

Accepted 28 June 2010

Available online 21 August 2010

\section{Keywords:}

Rett syndrome

Epilepsy

Genotype-phenotype correlation

Drug resistance

Risk factors

Seizure semiology

\begin{abstract}
A B S T R A C T
Epilepsy often occurs in Rett syndrome and is considered a major problem. The aim of this study was to define the clinical features of epilepsy and the correlation between seizures and both genotype and clinical phenotype in the Rett population. One hundred sixty-five patients with Rett syndrome referred to four Italian centers were recruited. All patients underwent video/EEG monitoring and molecular analysis of the MECP2 gene or, in negative cases, of the CDKL5 and FOXG1 genes. The frequency of epilepsy was 79\%. Drugresistant epilepsy occurred in 30\% of all our patients with Rett syndrome and in 38\% of those with epilepsy. Our findings demonstrate that epilepsy differs among the various phenotypes and genotypes with respect to age at onset, drug responsiveness, and seizure semiology. The Hanefeld and preserved speech variants represent the extremes of the range of severity of epilepsy: the preserved speech variant is characterized by the mildest epileptic phenotype as epilepsy is much less frequent, starts later, and is less drug resistant than what is observed in the other phenotypes. Another important finding is that seizure onset before 1 year of age and daily frequency are risk factors for drug resistance. Thus, this study should help clinicians provide better clinical counseling to the families of patients with Rett syndrome.
\end{abstract}

(c) 2010 Elsevier Inc. All rights reserved.

\section{Introduction}

Rett syndrome (RS) is a rare neurodevelopmental disorder affecting $1 / 10,000$ to $1 / 15,000$ females [1]. The diagnosis of RS is based on clinical characteristics, including an initial period of apparently normal development (ages 6-12 months), followed by a period of rapid decline with loss of hand use and spoken language and the onset of stereotypic hand movements, abnormal gait, and growth failure. The clinical spectrum varies from classic to variant forms, including formes frustes, which are characterized by preserved ambulation or speech and early onset or congenital presentation, with severe epilepsy [2]. In 1999, MECP2 gene mutations on the $\mathrm{X}$ chromosome were found to be associated with RS, and account for approximately $80 \%$ of cases [3, 4]. Recently, CDKL5 gene mutations have been identified in girls affected by atypical RS with early-onset seizures [5]. A third gene, FOXg1, is reported to be responsible for some congenital variant forms [6].

\footnotetext{
* Corresponding author. Department of Child Neuropsychiatry, Istituto "G. Gaslini," Largo Gaslini, 5, 16147 Genoa, Italy. Fax: +39 010381303.

E-mail address: mariapintaudi@hotmail.com (M. Pintaudi).
}

Although epilepsy occurs in about $80 \%$ of patients with RS [7], and despite being viewed both by families and by clinicians as a major problem in RS [8], there has been little research into the features of seizures and the correlation between genotype and epileptic phenotype [7, 9-11]. Both the presence and the severity of epilepsy have been correlated with low body mass index, early developmental problems, poorer mobility, and greater clinical severity [9, 11]. The effects of X-chromosome inactivation status and other epigenetic influences on gene expression are now being investigated, and the results suggest that one MECP2 target, that is, brain-derived neurotrophic factor (BDNF), plays a significant role in determining seizure severity in RS $[12,13]$.

The aim of this study was to define the clinical features of epilepsy in RS: age at seizure onset, semiology of seizures, and correlation between seizures and both genotype and clinical phenotype in the Rett population.

\section{Methods}

This multicenter, retrospective study was carried out between January 2008 and November 2009 at the Giannina Gaslini Children's 
Hospital, Genoa, Italy. To be eligible for this study, patients had to have been clinically diagnosed with Rett syndrome according to European Society of Paedriatic Neurology criteria defined in 2001; had to have undergone molecular analysis of the MECP2 gene or, in negative cases, of the CDKL5 gene; and had to have undergone video/EEG monitoring. On the basis of these criteria, data for 165 subjects were collected from a cohort of patients referred to four Italian child neuropsychiatry departments: G. Gaslini Institute, University of Genoa; St. Paolo Hospital, Milan; University Hospital, Siena; Spedali Civili, Brescia.

A questionnaire was made up in order to obtain longitudinal data including clinical information on seizures, EEG findings, and treatment. It was completed by one researcher, collecting data from patients'files, hospital charts and from the physician in charge of the patients. Phenotypes were categorized as follows: classic, congenital, preserved speech variant (PSV), formes frustees (FF), and early-onset seizure type (Hanefeld variant). The genotypes of cases with a previously identified MECP2 mutation were categorized as late truncating C-terminal deletions, large gene deletions, and $\mathrm{T} 158 \mathrm{M}$, R255X, R270X, R306C, R168X, R294X, R133C, R106W, and P152R mutations, and were then grouped into two categories: missense mutations and truncating mutations. As the importance of the MeCP2 protein in terms of functionality is well known, we divided the mutations into those involving (NLS+) and those not involving (NLS-) the nuclear localization signal (NLS) (Table 1).
Seizures were classified according to criteria of the International League Against Epilepsy [14]. We took into consideration seizure semiology at onset and at last follow-up. Each subject was scored with respect to frequency of seizures: $1=$ daily seizures, $2=$ weekly seizures, $3=$ monthly or sporadic seizures, $4=$ absence of seizures. An epileptic score was assigned to each patient: $1=$ no epileptic seizures, $2=$ drug-responsive epileptic seizures, $3=$ drug-resistant epileptic seizures. Microcephaly was also taken into consideration as a parameter in our investigation to identify the presence of a correlation with epilepsy. Drug-resistant epilepsy was defined as the failure of two appropriate AEDs, the occurrence of an average of one or more seizures per month for $\geq 18$ months, and no longer than a 3-month seizure-free hiatus during those 18 months [15].

The reasons for and methods of the study protocol were carefully explained to the families of the patients, and informed consent was obtained.

\subsection{Statistical analysis}

Data are described as means and SD or median and range for continuous variables, and as absolute and relative frequencies for categorical variables. Nonparametric analysis (Mann-Whitney $U$ test) for continuous variables and the Chi square or Fisher's exact test for categorical variables were used to measure differences between

Table 1

Characteristics of patients with Rett syndrome.

\begin{tabular}{|c|c|c|c|c|}
\hline Characteristic & All & No epilepsy & Drug-responsive epilepsy & Drug-resistant epilepsy \\
\hline$N$ & 165 & $35(21.2)$ & $81(49.1)$ & 49 (29.7) \\
\hline \multicolumn{5}{|l|}{ Phenotype } \\
\hline Classic forms & $140(84.8)^{\mathrm{a}}$ & $25(18)$ & $73(52)$ & $42(30)$ \\
\hline Preserved speech variant & $15(9.1)$ & $8(53.3)$ & $5(33.3)$ & $2(13.3)$ \\
\hline Formes frustes & $3(1.8)$ & $2(67)$ & $1(33)$ & - \\
\hline Hanefeld variant & $6(3.6)$ & - & $1(17)$ & $5(83)$ \\
\hline Congenital variant & $1(0.6)$ & - & $1(100)$ & - \\
\hline \multicolumn{5}{|l|}{ Genotype } \\
\hline MECP2+ & $147(89.1)$ & $30(20.4)$ & $75(51)$ & $42(28.6)$ \\
\hline CDKL5+ & $5(3)$ & - & $1(20)$ & $4(80)$ \\
\hline FOXG1+ & $1(0.6)$ & - & $1(100)$ & - \\
\hline Negative on genetic investigation & $12(7.3)$ & $5(41.7)$ & $4(33.3)$ & $3(25)$ \\
\hline \multicolumn{5}{|l|}{ Mecp2 mutations } \\
\hline Late truncating C-terminal deletions & $29(19.7)$ & $10(34.5)$ & $17(58.6)$ & $2(6.9)$ \\
\hline Large deletions & $10(6.8)$ & $1(10)$ & $7(70)$ & $2(20)$ \\
\hline T158M & $17(11.6)$ & $3(17.6)$ & $6(35.3)$ & $8(47.1)$ \\
\hline R255X & $16(10.9)$ & $2(12.5)$ & $7(43.8)$ & $7(43.8)$ \\
\hline R270X & $14(9.5)$ & $3(21.4)$ & $8(57.1)$ & $3(21.4)$ \\
\hline R306C & $14(9.5)$ & $2(14.3)$ & $8(57.1)$ & $4(28.6)$ \\
\hline R168X & $10(6.8)$ & $2(20)$ & $4(40)$ & $4(40)$ \\
\hline R294X & $9(6.1)$ & $1(11)$ & $4(44.5)$ & $4(44.5)$ \\
\hline $\mathrm{R} 133 \mathrm{C}$ & $6(4.1)$ & $2(33.3)$ & $3(50)$ & $1(16.7)$ \\
\hline R106W & $6(4.1)$ & - & $3(50)$ & $3(50)$ \\
\hline P152R & $5(3.4)$ & $1(20)$ & $3(60)$ & $1(20)$ \\
\hline Others $^{\mathrm{b}}$ & $11(7.5)$ & $3(27)$ & $5(46)$ & $3(27)$ \\
\hline \multicolumn{5}{|l|}{ Type of MECP2 mutation } \\
\hline \multicolumn{5}{|l|}{ Group $1^{\mathrm{c}}$} \\
\hline Missense & 54 & $10(18.5)$ & $25(46.3)$ & $19(35.1)$ \\
\hline Truncating & 54 & $9(16.6)$ & $26(48.2)$ & $19(35.1)$ \\
\hline \multicolumn{5}{|l|}{ Group 2} \\
\hline NLS- & 84 & $20(23.8)$ & $42(50)$ & $22(26.2)$ \\
\hline NLS+ & 63 & $10(15.9)$ & $33(52.3)$ & $20(31.7)$ \\
\hline Microcephaly & $96(60.4)$ & $17(51.5)$ & $51(65.4)$ & $28(58.3)$ \\
\hline \multicolumn{5}{|l|}{ Age at epilepsy onset } \\
\hline Mean $\pm S D$ & $4.7 \pm 3.7$ & - & $5.3 \pm 4.2$ & $3.6 \pm 2.5^{\mathrm{d}}$ \\
\hline Median (range) & $4(0.1-21)$ & & $4(0.1-21)$ & $3.2(0.1-12)$ \\
\hline \multicolumn{5}{|l|}{ Age at last follow-up } \\
\hline Mean \pm SD & $14.9 \pm 8.5$ & $13.7 \pm 10.4$ & $15 \pm 7.9$ & $16 \pm 8.2$ \\
\hline Median (range) & $14(2-40)$ & $14(3-37)$ & $14(2-37)$ & $15(3-40)$ \\
\hline
\end{tabular}

a $N(\%)$.

b Includes one patient for each of the following mutations: E14fs, P251fs, P302L, Q297X R111G, R306H, R453X, T322A, L124F, Y141X, D156E.

c Group 1 does not include late truncanting C-terminal deletions and large deletions.

d Drug-resistant epilepsy versus drug-responsive epilepsy, $P=0.02$. 
groups. $P$ values $\leq 0.05$ were considered statistically significant, and all $P$ values were based on two-tailed tests. Statistical analysis was performed using SPSS for Windows (SPSS Inc., Chicago, IL, USA).

\section{Results}

The clinical features of our series of 165 patients are detailed in Table 1 . The median age at last follow up was 14 years (range: $2-40$ ).

There were $140(84.8 \%)$ patients with classic forms, 15 (9.1\%) with the PSV, 6 (3.6\%) with the Hanefeld variant, 3 (1.8\%) with formes frustes, and 1 (0.6) with the congenital form. Eight patients with classic forms were negative on genetic investigation and 132 were found to carry an MECP2 mutation. Fourteen patients with the PSV had a mutation in the MECP2 gene. All patients with forma frusta were negative on genetic investigation. Patients with the Hanefeld variant included all five subjects with CDKL5 mutations and one subject with a MECP2 mutation (R106W). The patient with the congenital form had a mutation in the FOXG1 gene.

There were 130 (79\%) patients with epilepsy in our cohort, and their median age at last follow-up was 14.5 years (range: $2-40$ ); 37 (28.5\%) were $\geq 20$ years of age. The median age at epilepsy onset was 4 years (range: 1.5 months to 21 years). Seizures started after 10 years of age in 10 (7.7\%) patients. Epileptic seizures always occurred in those with the Hanefeld variant, whereas they occurred in $82 \%$ of patients with classic forms but in only $46.7 \%$ of those with the PSV $(P=0.004)$. Patients with the PSV started having seizures later in life than those with classic forms ( $6.1 \pm 4.3$ years vs $4.8 \pm 3.6$ years, $P=0.01$ ).

With respect to genotype, all patients with CDKL5 mutations started having seizures within the first 12 months of life $(0.45 \pm$ 0.49 years), whereas the mean age at seizure onset was 4.7 years for patients with an MECP2 mutation and 6.6 years for patients without any identified mutation. Among our patients with MECP2 mutations, we observed that subjects with large gene deletions started having seizures later $(6.72 \pm 7.14$ years) than those with the other types of mutations, particularly those with R168X and R255X mutations $(3.12 \pm 1.86$ and $3.9 \pm 2.0$ years, respectively).

There were no differences in age at seizure onset between those with NLS+ mutations and those with NLS- mutations ( $4.5 \pm 3.8$ years vs $4.9 \pm 3.5$ years, $P=0.46$ ).

Patients were subdivided into three groups on the basis of epileptic score: patients without epileptic seizures $(n=35,21.2 \%)$, patients with drug-responsive epileptic seizures ( $n=81,49.1 \%$ ), and patients with drug-resistant epileptic seizures ( $n=49,29.7 \%$ ) (Table 1$)$. There were no significant differences among the groups with respect to age at last follow-up.

We observed that of the 37 patients who were $\geq 20$ years of age, 25 (67.5\%) were drug responsive and 12 (32.5\%) were drug resistant, whereas in the group $<20$ years of age, 56 (60\%) subjects were drug responsive. Eighty percent of patients with late-onset seizures (after 10 years of age) were drug responsive and had monthly or sporadic seizures.

With respect to the correlation between epileptic score and clinical phenotype, we found that drug-resistant epilepsy occurred significantly more often in those with the Hanefeld variant than in those with classic forms ( $83 \%$ vs $30 \%, P=0.03$ ). This difference was also observed between patients with the Hanefeld variant and those with the PSV (13\%), but it did not reach statistical significance, most likely because of the small number of patients (Fig. 1). The mean age at seizure onset differed significantly between patients with drugresponsive seizures $(5.3 \pm 4.2$ years $)$ and those with drug-resistant seizures $(3.6 \pm 2.5$ years, $P=0.02)$.

Moreover, monthly or sporadic seizures started at an older age as compared with daily seizures ( $5.8 \pm 5.3$ years vs $3.1 \pm 2.2$ years, $P=0.02$ ). Thus, we observed that an age at epilepsy onset $\leq 1$ year and daily frequency at onset represent risk factors for drug resistance in

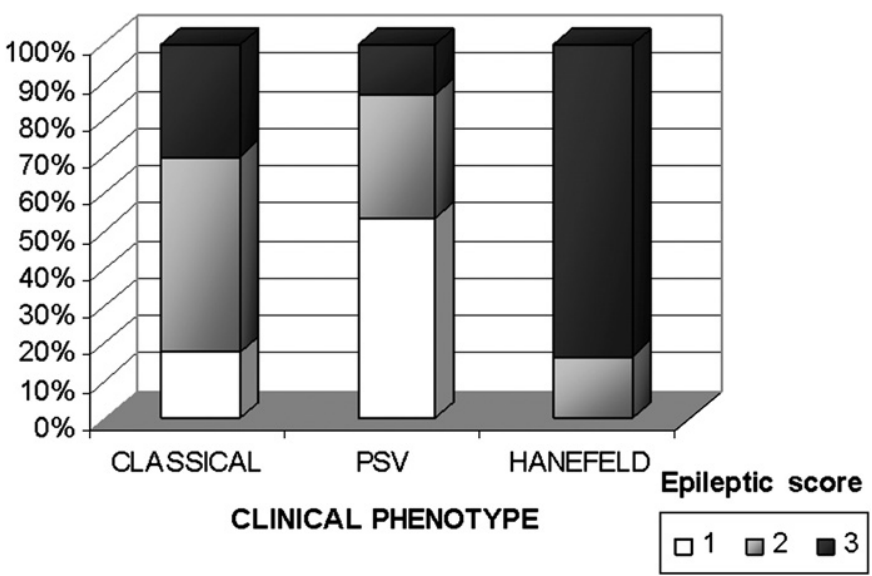

Fig. 1. Epileptic score versus phenotype. Epileptic score: $1=$ no epileptic seizures, $2=$ drug-responsive epileptic seizures, $3=$ drug-resistant epileptic seizures.

patients with Rett syndrome with epilepsy $(\mathrm{OR}=4.27,95 \% \mathrm{CI}=1.23$ $14, P=0.02 ; \mathrm{OR}=6.24,95 \% \mathrm{CI}=1.90-20, P=0.009$ ) (Table 2 ).

To statistically evaluate the correlation between epilepsy and genotype, we could include only patients with no mutations and those with the most frequently found mutations, that is, late truncating C-terminal deletions, large gene deletions, and T158M, R255X, R270X, R306C, R168X, and R294X mutations. Epilepsy occurred more frequently in patients with large gene deletions $(N=9 / 10,90 \%)$ and the R294X mutation $(N=8 / 9,89 \%)$, and less frequently in subjects with no identified mutations $(N=7 / 12,58 \%)$ and C-terminal deletions ( $N=19 / 29,66 \%$ ). Moreover, C-terminal deletions seem to be a protective factor compared with the other mutations with respect to both the occurrence of epilepsy and drug resistance $(\mathrm{OR}=0.43,95 \%$ $\mathrm{Cl}=0.16-1.13, P=0.054$, and $\mathrm{OR}=0.16,95 \% \mathrm{Cl}=0.02-0.78, P=0.02$, respectively). In particular, C-terminal deletions are a protective factor for drug-resistant epilepsy as compared with the most frequent mutations: $\mathrm{R} 255 \mathrm{X}(\mathrm{OR}=0.12,95 \% \mathrm{CI}=0.01-0.89, P=0.02)$; $\mathrm{T} 158 \mathrm{M}$ $(\mathrm{OR}=0.09,95 \% \mathrm{Cl}=0.01-0.67, P=0.007), \mathrm{R} 294 \mathrm{X} \quad(\mathrm{OR}=0.12,95 \%$ $\mathrm{CI}=0.01-1.19, P=0.04)$.

Of the 52 patients known to have $X$ inactivation, 33 had balanced $X$ inactivation. No significant correlations were found with either the presence or severity of epilepsy.

We observed that microcephaly was more frequent in patients with classic forms (65\%) than in those with the PSV and Hanefeld variant (21.4 a d 33.3\%, $P=0.01$ ). Microcephaly occurred more frequently in patients carrying NLS+ mutations (80\%) than in those carrying NLS- mutations $(51 \%)(P=0.001)$. No significant correlation

Table 2

Clinical risk factors for drug-resistant epilepsy: Univariate analysis.

\begin{tabular}{|c|c|c|c|c|c|}
\hline Characteristic & $\begin{array}{l}\text { Drug-responsive } \\
\text { epilepsy }\end{array}$ & $\begin{array}{l}\text { Drug-resistant } \\
\text { epilepsy }\end{array}$ & OR & $95 \% \mathrm{CI}$ & $P$ value \\
\hline \multicolumn{6}{|c|}{ Age at epilepsy onset } \\
\hline$>1$ year & $76(95)$ & $39(81.3)$ & Ref. & & \\
\hline$\leq 1$ year & $4(5)$ & $9(18.8)$ & 4.27 & $1.23-14$ & 0.02 \\
\hline \multicolumn{6}{|l|}{ Microcephaly } \\
\hline No & $27(34.6)$ & $20(41.7)$ & & & \\
\hline Yes & $51(65.4)$ & $28(58.3)$ & 0.74 & $0.35-1.55$ & 0.43 \\
\hline \multicolumn{6}{|c|}{ Seizure semiology } \\
\hline $\begin{array}{l}\text { Complex partial } \\
\text { seizures }\end{array}$ & $36(46.2)$ & $20(44.4)$ & Ref. & & \\
\hline $\begin{array}{l}\text { Generalized } \\
\text { seizures }\end{array}$ & $37(47.4)$ & $41(51.1)$ & 1.12 & $0.53-2.38$ & \\
\hline Both & $5(6.4)$ & $2(4.4)$ & 0.72 & $0.13-4.06$ & 0.87 \\
\hline \multicolumn{6}{|c|}{ Seizure frequency at onset } \\
\hline Monthly & $23(47.9)$ & $5(16.7)$ & Ref. & & \\
\hline Weekly & $11(22.9)$ & $6(20)$ & 2.51 & $0.63-10$ & \\
\hline Daily & $14(29.2)$ & $19(63.3)$ & 6.24 & $1.90-20$ & 0.009 \\
\hline
\end{tabular}


was found with either the presence or the severity of epilepsy. ( $P=0.33$ and $P=0.37$, respectively).

Data concerning the semiology of seizures at onset were available for 123 patients (Table 3). Complex partial seizures (CPS) occurred in 56 patients (45.5\%), and generalized seizures (GS) in 60 subjects (48.8\%). Seven patients (5.7\%) had both kinds of seizures: three patients had CPS and generalized tonic-clonic seizures [GTCS], two patients had CPS and atypical absences, one patient had CPS and myoclonic seizures, and 1 patient had CPS and spasms). Among 56 patients with partial seizures, 30 (53\%) had a secondary generalization. Five patients had two kinds of generalized seizures: one had atypical absences and spasms; one had GTCS and atypical absences; one had atonic and tonic seizures; one had GTCS, atonic, and tonic seizures; and one had atypical absences and tonic seizures. Generalized seizure semiology is illustrated in Fig. 2.

Because of the peculiarity of the CDKL5 epileptic phenotype $[5,16-$ 20] we also studied it as a distinct category: seizures at onset were generalized in two patients (one with GTCS and one with spasms) and partial in two other cases (both with generalization), whereas one patient had partial seizures and spasms. There were no significant differences in seizure semiology at onset between patients with and those without the MECP2 mutation or among the patients with different kinds of mutations.

As for drug responsiveness, there were no differences between patients with partial and those with generalized seizures $(64.3 \%$ vs $61.7 \%$, respectively) at onset. At follow-up, seizure semiology was known for 92 subjects (Table 3). Generalized seizure semiology was similar to what it was at seizure onset. Among the patients with CDKL5 mutations, two still had generalized seizures (one with myoclonic seizures and the other with myoclonic seizures, spasms, and GTCS), whereas spasms and partial seizures occurred in two cases, and GTCS in another case.

Patients with the Hanefeld variant more frequently presented with spasms (50\%) and myoclonic seizures (25\%) than those with the other phenotypes ( $7 \%$ and $6 \%$, respectively).

Partial seizures responded to drugs more frequently than generalized seizures (65.2\% vs $48.7 \%$, respectively), but this difference was not statistically significant $(P=0.22)$.

Physicians reported epileptic status in only 11 of our cases; however, no additional data are available.

\section{Discussion}

In this study we analyzed the clinical features of epilepsy in RS, particularly with respect to age at seizure onset, semiology of seizures, and correlation with genotype and clinical phenotype. The frequency of epilepsy among our cases was about $80 \%$. This is in agreement with data from the largest reports, such as the Australian study [7, 9].
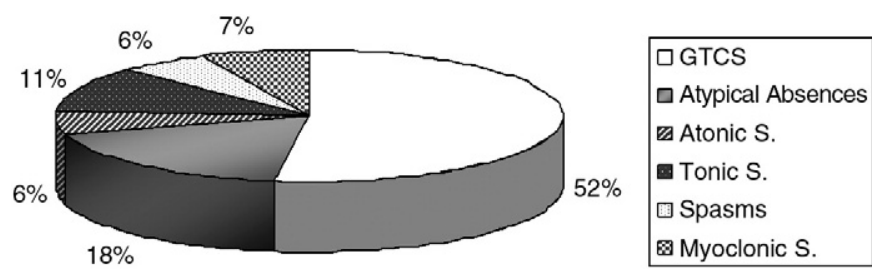

Fig. 2. Generalized seizure semiology at onset.

Most seizures among our patients started between 2 and 5 years of age. Only a very small percentage of our patients had seizure onset after 10 years of age, and epilepsy was mild in most of these patients, with sporadic frequency and drug-responsive seizures. We observed similar percentages of partial and generalized seizures at onset, and GTCS were the most frequent. At their last follow-up, most patients were still having one kind of seizure, and GTCS were again the most frequent, followed by atypical absences.

Our data show that semiology of seizures is not significantly correlated with severity of epilepsy, even if generalized seizures would appear to be more often associated with drug-resistant epilepsy, as reported by Steffenburg et al. [21].

As the role of head circumference growth in association with epilepsy is not clear [9], we investigated the correlation between epilepsy and microcephaly and found that microcephaly is more frequent in patients with classic forms than in those with the PSV or Hanefeld variant. We also found that it is more frequent in patients carrying NLS+ mutations than in those with NLS- mutations, but, unlike Steffenburg et al. [21] and Moser et al. [22], we found no correlation with either the presence or the severity of epilepsy. This finding is similar to that reported by Glaze et al. in the American cohort [11].

With respect to clinical phenotype, we found that the PSV is significantly correlated with a lower incidence of epilepsy than classic forms. In people with the PSV, seizures start later and are drug responsive in most cases. Thus, epileptic features of the PSV are milder than classic phenotypes, as has been observed for various clinical parameters reported by other authors [23, 24]. No differences were found in seizure semiology among the clinical phenotypes at seizure onset or at last follow-up, except for patients with the Hanefeld variant, who more frequently had spasms and myoclonic seizures as compared with patients with the other phenotypes.

In most cases (83\%), the Hanefeld variant was associated with a CDKL5 mutation, and it has been confirmed as being the form with the most severe epileptic phenotype [5, 16-20].

We found that mutations of the CDKL5 gene were always correlated with early-onset epilepsy, and that all patients had seizures within 12 months, whereas patients with no identified mutations had

Table 3

Semiology of seizures.

\begin{tabular}{|c|c|c|c|c|c|c|}
\hline \multirow[t]{2}{*}{ Characteristic } & \multicolumn{3}{|l|}{ At onset } & \multicolumn{3}{|l|}{ At follow-up } \\
\hline & Complex partial seizures & Generalized seizures & Both & Complex partial seizures & Generalized seizures & Both \\
\hline$N$ & $56(45.5)^{\mathrm{a}}$ & $60(48.8)$ & $7(5.7)$ & $24(26.1)$ & $39(42.4)$ & $29(31.5)$ \\
\hline \multicolumn{7}{|l|}{ Phenotype } \\
\hline Classic forms & $50(46.3)$ & $53(49.1)$ & $5(4.6)$ & $22(27.2)$ & $34(42)$ & $25(30.9)$ \\
\hline Preserved speech variant & $2(28.6)$ & $4(57.1)$ & $1(14.3)$ & & $3(75 \%)$ & $1(25)$ \\
\hline Formes frustes & & $1(100)$ & & $1(100)$ & & \\
\hline Hanefeld variant & $3(50)$ & $2(33.3)$ & $1(16.7)$ & $1(16.7)$ & $2(33.3)$ & $3(50)$ \\
\hline Congenital variant & $1(100)$ & & & & & \\
\hline \multicolumn{7}{|l|}{ Genotype } \\
\hline MECP2+ & $50(45.5)$ & $55(50)$ & $5(4.5)$ & $21(26)$ & $37(46)$ & $23(28)$ \\
\hline CDKL5+ & $2(40)$ & $2(40)$ & $1(20)$ & - & $2(40)$ & $3(60)$ \\
\hline FOXG1+ & $1(100)$ & & & - & - & - \\
\hline Negative on genetic investigations & $3(42.9)$ & $3(42.9)$ & $1(14.3)$ & $3(50)$ & - & $3(50)$ \\
\hline
\end{tabular}


a much lower seizure frequency than other groups and their seizures started later. The latter point differs from what was reported by the Australians [7], probably because our group of patients with no mutations did not include any patients with CDKL5 mutations, whereas the Australian study most likely did.

In patients with MECP2 mutations, $\mathrm{C}$-terminal deletions proved to be a protective factor as compared with the remaining Rett population, with respect to both the occurrence of epilepsy and drug resistance. At this point it is important to consider that a specific BDNF polymorphism may influence epileptic phenotype and drug responsiveness [12,13]. Therefore, further genetic analysis is being carried out in our patients.

Drug-resistant epilepsy occurred in 30\% of all our patients with RS and in $38 \%$ of those with RS with epilepsy. These data show a higher percentage of drug resistance than what was reported by Buoni et al. [25]. Nonetheless, they confirm that most cases of Rett syndrome with epilepsy are not drug resistant. Patients with drug-resistant epilepsy start having seizures significantly earlier than subjects with drugresponsive epilepsy $(3.6 \pm 2.5$ years vs $5.3 \pm 4.2$ years, $P=0.02)$, and their seizures are more frequent, often occurring daily.

We observed that both seizure onset before 1 year of age and daily frequency are risk factors for drug resistance. Moreover, unlike Steffenburg et al. [21], we observed no significant differences in drug responsiveness between patients with epilepsy $\geq 20$ years of age and younger subjects. This could mean that drug-resistant epilepsy often continues, even at a later age.

In conclusion, epilepsy in Rett syndrome differs among the various phenotypes and genotypes with respect to age at onset, drug responsiveness, and seizure semiology. CDKL5 mutations are always correlated with early-onset epilepsy, which is drug resistant in most cases. About $80 \%$ of patients with MECP2 mutations have seizures, with a $28 \%$ rate of drug resistance. Patients with no identified mutations have the lowest prevalence of epileptic seizures and the latest onset. Taking into consideration the phenotypes, except for the Hanefeld variant, which is associated with the highest frequency of myoclonic seizures and spasms, we observed similar percentages of cases having partial and generalized seizures in both patients with classic forms and those with the PSV, with GTCS being the most frequent type. Compared with the other phenotypes, the PSV is characterized by mild epilepsy, that is, less frequent seizures, later onset, and less drug resistance.

The most clinically relevant points of this study include the definition of epileptic features for various phenotypes and genotypes of Rett syndrome and the improvement of our prognostic knowledge of epilepsy, thus providing the basis for clinical counseling for the families of patients with Rett syndrome.

\section{References}

[1] Hagberg B, Hagberg G. Rett syndrome: epidemiology and geographical variability. Eur Child Adolesc Psychiatry 1997;6:S5-7.

[2] Hagberg B, Hanefeld F, Percy A, Skjeldal O. An update on clinically applicable diagnostic criteria in Rett syndrome. Comments to Rett Syndrome Clinical Criteria Consensus Panel Satellite to European Paediatric Neurology Society Meeting, Baden Baden, Germany, 11 September 2001. Eur J Paediatr Neurol 2002;6:293-7.

[3] Amir RE, Van den Veyver IB, Wan M, Tran CQ, Francke U, Zoghbi HY. Rett syndrome is caused by mutations in X-linked MECP2, encoding methyl-CpG-binding protein 2. Nat Genet 1999;23:185-8.

[4] Bienvenu T, Carrie A, De Roux N, et al. MECP2 mutations account for most cases of typical forms of Rett syndrome. Hum Mol Genet 2000;9:1377-84

[5] Scala E, Ariani F, Mari F, et al. CDKL5/STK9 is mutated in Rett syndrome variant with infantile spasms. J Med Genet 2005;42:103-7.

[6] Ariani F, Hayek G, Rondinella D, et al. FOXG1 is responsible for the congenital variant of Rett syndrome. Am J Hum Genet 2008;83:89-93.

[7] Jian L, Nagarajan L, de Klerk N, et al. Predictors of seizure onset in Rett syndrome. J Pediatr 2006;149:542-7.

[8] Bahi-Buisson N, Guellec I, Nabbout R, et al. Parental view of epilepsy in Rett syndrome. Brain Dev 2008;30:126-30.

[9] Jian L, Nagarajan L, de Klerk N, Ravine D, Christodoulou J, Leonard H. Seizures in Rett syndrome: an overview from a one-year calendar study. Eur J Paediatr Neurol 2007;11:310-7.

[10] Huppke P, Köhler K, Brockmann K, Stettner GM, Gärtner J. Treatment of epilepsy in Rett syndrome. Eur J Paediatr Neurol 2007;11:10-6.

[11] Glaze DG, Percy AK, Skinner S, et al. Epilepsy and the natural history of Rett syndrome. Neurology 2010;74:909-12.

[12] Nectoux J, Bahi-Buisson N, Guellec I, et al. The p.Val66Met polymorphism in the BDNF gene protects against early seizures in Rett syndrome. Neurology 2008;70: 2145-51.

[13] Zeev BB, Bebbington A, Ho G, et al. The common BDNF polymorphism may be a modifier of disease severity in Rett syndrome. Neurology 2009;72:1242-7.

[14] Engel J, for the International League against Epilepsy ILAE. A proposed diagnostic scheme for people with epileptic seizures and with epilepsy: report of the ILAE Task Force on Classification and Terminology. Epilepsia 2001;42:796-803.

[15] Berg AT, Kelly MM. Defining intractability: comparisons among published definitions. Epilepsia 2006;47:431-6.

[16] Bahi-Buisson N, Nectoux J, Rosas-Vargas H, et al. Key clinical features to identify girls with CDKL5 mutations. Brain 2008;131:2647-61.

[17] Bahi-Buisson N, Kaminska A, Boddaert N, et al. The three stages of epilepsy in patients with CDKL5 mutations. Epilepsia 2008;49:1027-37.

[18] Pintaudi M, Baglietto MG, Gaggero R, et al. Clinical and electroencephalographic features in patients with CDKL5 mutations: two new Italian cases and review of the literature. Epilepsy Behav 2008;12:326-31.

[19] Evans JC, Archer HL, Colley JP, et al. Early onset seizures and Rett-like features associated with mutations in CDKL5. Eur J Hum Genet 2005;13:1113-20.

[20] Artuso R, Mencarelli MA, Polli R, et al. Early-onset seizure variant of Rett syndrome: definition of the clinical diagnostic criteria. Brain Dev 2010;32:17-24.

[21] Steffenburg U, Hagberg G, Hagberg B. Epilepsy in a representative series of Rett syndrome. Acta Paediatr 2001;90:34-9.

[22] Moser SJ, Weber P, Lütschg J. Rett syndrome: clinical and electrophysiologic aspects. Pediatr Neurol 2007;36:95-100.

[23] Kerr AM, Archer HL, Evans JC, Prescott RJ, Gibbon F. People with MECP2 mutationpositive Rett disorder who converse. J Intellect Disabil Res 2006;50:386-94.

[24] Renieri A, Mari F, Mencarelli MA, et al. Diagnostic criteria for the Zappella variant of Rett syndrome (the preserved speech variant). Brain Dev 2009;31:208-16.

[25] Buoni S, Zannolli R, Felice CD, et al. Drug-resistant epilepsy and epileptic phenotype-EEG association in MECP2 mutated Rett syndrome. Clin Neurophysiol 2008;119:2455-8. 